\title{
Interventional pulmonology during COVID-19 pandemic: current evidence and future perspectives
}

\author{
Roberto Piro, Eleonora Casalini, Francesco Livrieri, Matteo Fontana, Giulia Ghidoni, Sofia Taddei, \\ Nicola Facciolongo
}

Pulmonology Unit, Azienda Unità Sanitaria Locale - IRCCS di Reggio Emilia, Italy

Contributions: (I) Conception and design: R Piro, N Facciolongo; (II) Administrative support: R Piro, N Facciolongo; (III) Provision of study materials or patients: R Piro, E Casalini, F Livrieri, M Fontana, G Ghidoni; (IV) Collection and assembly of data: R Piro, E Casalini, F Livrieri, M Fontana, G Ghidoni; (V) Data analysis and interpretation: R Piro, E Casalini, N Facciolongo; (VI) Manuscript writing: All authors; (VII) Final approval of manuscript: All authors.

Correspondence to: Roberto Piro, MD. Pulmonology Unit, Azienda Unità Sanitaria Locale - IRCCS di Reggio Emilia, Via Amendola, 242122 Reggio Emilia, Italy. Email: roberto.piro@ausl.re.it.

\begin{abstract}
COVID-19, caused by SARS-CoV-2 infection, has become increasingly prevalent worldwide, reaching a pandemic stage in March 2020. The organization of health care services had to change because of this new disease, with the need to reallocate staff and materials, besides changing management protocols. A very important challenge is not to expose patients and health care workers to the risk of infection and not to waste personal protective equipment (PPE). In the field of interventional pulmonology, various aspects related to COVID-19 must be taken into great consideration. Although bronchoscopy is not a first-line test for patients with suspected SARS-CoV-2 infection, it has a role in selected cases and it can be useful for differential diagnosis. However, bronchoscopy is an aerosol-generating procedure, that's why its unjustified use could contribute to propagate the virus. For this reason, the utility of each procedure must be carefully evaluated, the patient has to be properly investigated before the procedure, which has to be performed with specific precautions, including adequate PPE. In this review, we summarize the knowledge and the principal statements about endoscopic activity in COVID-19 period, in both diagnosis of COVID-19 and management of patients. How to safely perform both bronchoscopic and pleural-related procedures (thoracoscopy, pleural biopsy and drainage of pleural effusions) is described with the aim to help the staff to decide when and how performing a procedure. We also highlight how interventional pulmonology could help in matter of complications related to COVID-19.
\end{abstract}

Keywords: Thoracoscopy; bronchoscopy; interventional pulmonology; COVID-19; SARS-CoV-2

Submitted Jun 13, 2020. Accepted for publication Feb 07, 2021.

doi: $10.21037 /$ jtd-20-2192

View this article at: http://dx.doi.org/10.21037/jtd-20-2192

\section{Introduction}

In early January 2020, a novel coronavirus, Severe Acute Respiratory Syndrome Coronavirus 2 (SARS-CoV-2), was identified as the cause of the increasing in pneumonia cases reported in Wuhan (1). This finding emerged from the analysis of bronchoalveolar washing fluid of a patient (2). Due to its person-to-person transmission, coronavirus disease rapidly spread worldwide (3), becoming a pandemic. On 11 February 2020, the World Health Organization named the SARS-CoV-2-induced illness as 2019 coronavirus disease (COVID-19). As of 19 January 2021, over 93,000,000 COVID-19 cases have been reported around the world, with over 2,000,000 deaths (4).

The main symptoms of SARS-CoV-2 infection are fever, asthenia, dry cough, dyspnea ageusia and anosmia (5). In some cases, severe interstitial pneumonia, acute respiratory distress syndrome (ARDS) and subsequent multiorgan 
failure are responsible for severe acute respiratory failure and high death rate associated to this infection.

Since SARS-CoV-2 is predominantly transmitted via droplets and fomites, healthcare workers (HCW) participating in aerosol-generating procedures (such as bronchoscopy, endotracheal intubation (ETI), upper gastrointestinal endoscopy, otolaryngologic procedures involving the upper airway, tracheotomy) are at high risk of infection (6). Considering the relevance of the topic, few days after the beginning of the pandemic several societies of interventional pulmonology provided guidelines regarding bronchoscopy during COVID-19 pandemic (7-9). In this review we analyze and summarize the suggested behavior about endoscopic activity in COVID-19 period, derived from statements of scientific societies and articles that has been already published in literature. PubMed was searched for publications about COVID-19 and bronchoscopy or thoracoscopy; websites of the main international scientific societies were also consulted. We conducted thematic synthesis of the results of the included publications. Particularly, we analyze in which cases interventional pulmonology can be useful in the management of COVID-19 patients with acute clinical problems related to it; furthermore, we describe how to manage endoscopic procedures in the COVID-era focusing on the timing and how performing them in both areas of bronchoscopy and pleural procedures. In addition to this, we explore the future perspectives of interventional pulmonology in the post pandemic era. We present the following article in accordance with the Narrative Review reporting checklist (available at http://dx.doi.org/10.21037/jtd-20-2192).

\section{Diagnosis of COVID-19}

In this pandemic period, the diagnosis is needed as quickly as possible, therefore it is common to collect a variety of upper and lower respiratory samples including throat, nasal, nasopharyngeal (NP), sputum and bronchial fluid (10-12). RT-PCR is a test that allows the qualitative determination of the virus nucleic acids (RNA) and is commonly used to search for the presence of the virus in the upper airways. SARS-CoV-2 RNA detected in oropharyngeal (OP) swabs is lower $(32 \%)$ than that in NP swabs $(63 \%)(13)$. The sensitivity on bronchoalveolar lavage (BAL), brush biopsy and sputum resulted respectively $93 \%, 46 \%$ and $72 \%$ (13).

NP swabs are currently performed for rapid and qualitative detection of SARS-CoV-2 antigen, in particular with the purpose of mass screening considering its many advantages, such as its low cost and rapidity, but it is characterized by a low sensitivity, especially when the viral load is not high $(14,15)$.

Several studies addressed the importance of chest computed tomography (CT) examination in COVID-19 patients (16) and reported the CT sensitivity as 98\% (17). Additionally, CT examination is also important in monitoring disease progression and evaluating therapeutic efficacy, especially in epidemic areas with high pre-test probability for the disease $(18,19)$. The positive and negative predictive value of chest CT for COVID-19 are estimated at $92 \%$ and $42 \%$ respectively, in a population with high pretest probability for the disease (19-21).

Antibody tests are not able to show if the patient has a current infection, because it can take 1-3 weeks after infection to make antibodies (22). However, serology can facilitate the diagnosis of SARS-CoV-2 infections when an NP swab specimen was collected inappropriately and the molecular assays were performed unsatisfactorily (23). Their main use is for epidemiological scope. Rapid serological antigenic tests (for IgG and IgM detection) are also available for mass screening in asymptomatic patients, but they are not indicated for clinical diagnosis (24).

\section{The role of interventional pulmonology in COVID-19 patients}

\section{Bronchoscopy for the diagnosis of SARS-CoV-2 infection}

Bronchoscopy is an invasive procedure achieved in different settings and with heterogeneous characteristics between performing centers (25). According with current statements $(8,9,26)$, this exam should not be a first-line test for patients with suspected COVID-19 infection. This infection is rather considered a relative contraindication to airway endoscopy and it is discouraged (8). Even if finding the virus RNA in the BAL has a specificity that CT cannot offer, the estimated sensitivity of BAL (93\%) is not higher than CT (13). Moreover, a recent Italian multicentric retrospective study evaluated 79 patients who underwent bronchoscopy with $\mathrm{BAL}$, with at least one negative or indeterminate nasal or NP swab considering the peristence of clinical suspect for COVID-19. This study releved only two patients with negative swabs and a positive detection of SARSCoV-2 RNA in BAL, supporting a limited role for BAL in diagnosis of COVID-19 if thoracic imaging and upper respiratory tract specimens are concordantly negative (27). The advantage of using bronchoscopy to confirm the diagnosis of COVID-19 and to detect co-infection or virus- 
Role of bronchoscopy in COVID-19 era

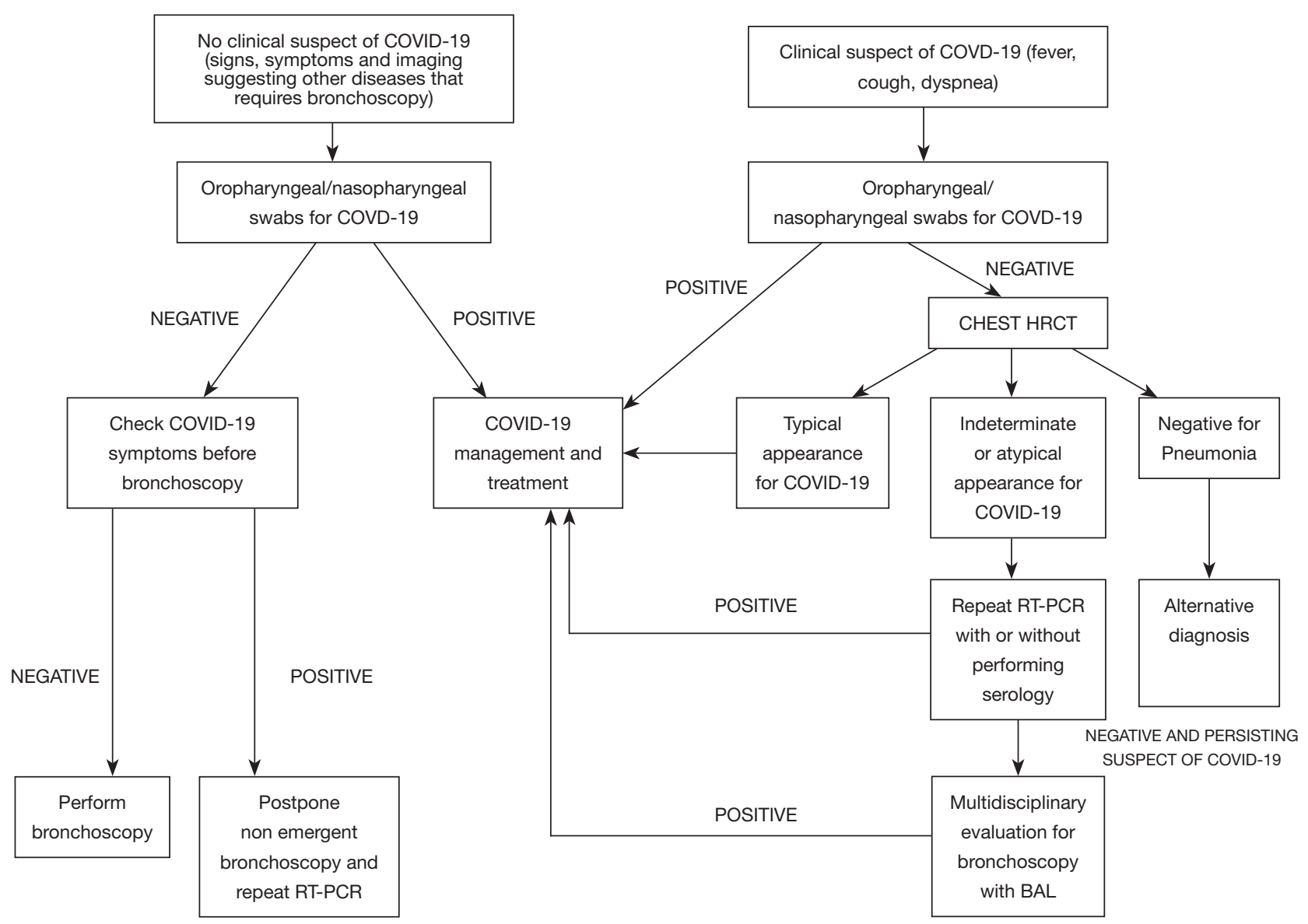

Figure 1 Proposal of flow-chart about the role of bronchoscopy in COVID-19 era.

related complications has to be carefully weighed because of the risk of bronchoscopy in propagating the infection (28). One study demonstrated that SARS-CoV-2 could remain aerosolized for up to 3 hours in experimental conditions (29). Furthermore, it should also be remembered that BAL is a procedure that can worsen the patient's respiratory failure.

Nevertheless, a survey conducted in Italy has revealed that bronchoscopies for diagnosing COVID-19 are quite frequent, both in intubated and non-intubated patients (30). Since this use of bronchoscopy is not in line with international recommendations $(8,9,26)$, the Italian Thoracic Society - Associazione Italiana Pneumologi Ospedalieri (ITS-AIPO) recommended avoiding unnecessary bronchoscopies, proposing a diagnostic algorithm that involves performing a swab and, in case of negativity, a chest CT scan and a pneumological assessment (30).
If the presence of COVID-19 is excluded, consequent measures will be taken; in the event that the pattern is typical or at least probable, the patient must be managed as affected by COVID-19. Only if the patient does not fall into any of the two previous cases and the SARS$\mathrm{CoV}-2$ infection is possible, a multidisciplinary evaluation (pulmonologist, infectious disease specialist, radiologist) should be performed to evaluate the indication for BAL (30); in this way, COVID-19 can be confirmed or an alternative diagnosis could be obtained (31). A proposal of flow-chart about the role of bronchoscopy in this field is reported in Figure 1. When bronchoscopy is considered necessary, a collection of a sample (minimum of $2-3 \mathrm{~mL}$ ) into a sterile and leak proof container is recommended and it is suggested to alert laboratory personnel regarding COVID-19 specimen processing and testing (8). 


\section{Bronchoscopy in the management of COVID-19}

The management of patients with COVID-19 is complex and bronchoscopy may be helpful under some circumstances, such as tracheobronchial obstruction by secretions (for example in the case of bacterial superinfection and/or ventilator-associated pneumonia) or the appearance of hemoptysis. The latter can be favored by the use of anticoagulant drugs often used in the management of these patients $(32,33)$. Bronchoscopy can also be useful in tracheostomized patients with COVID-19 and it can be functional to perform tracheobronchial toilets, to search for complications related to tracheostomy or to carry out a preliminary evaluation for tracheostomy tube removal $(34,35)$. Another emergent role of bronchoscopy in COVID-19 patients is for the diagnosis of COVID-19 associated pulmonary aspergillosis (CAPA). It is defined as invasive pulmonary aspergillosis (IPA) in temporal proximity to a preceding SARS-CoV-2 infection (36). Increasing number of reports documenting CAPA cases raised concerns about this superinfection as an additional contributing factor to mortality $(37,38)$. Patients with respiratory insufficiency requiring intensive care are at high risk for CAPA; the risk is further increased by corticosteroids or anti-IL-6 receptors treatments. Recently, the European Confederation for Medical Mycology and the International Society for Human and Animal Mycology instituted a group of experts to propose consensus criteria for the definition of CAPA (36); three different grades were proposed distinguishing in proven, probable and possible CAPA. CAPA can be also distinguished in pulmonary or tracheobronchial infection. Tracheobronchitis can be defined only by the direct visualisation via bronchoscopy of alterations in tracheobronchial tree as ulceration, nodule, pseudomembrane, plaque or eschar. In addition, bronchoscopy allows to collect respiratory specimens from the lower respiratory tract for microbiological tests; in particular, an infection biomarker such as galactomannan is validated for BAL but not for other specimens, including tracheal aspirate and non-bronchoscopic lavage. Lung biopsy, usually contemplated for diagnosis of IPA, is a procedure with a high-risk of complications in this population and for this reason it is scarcely used.

In all these circumstances, the choice to perform bronchoscopy must be made by carefully evaluating both risks of the procedure and risks of not performing it, even danger from alternative interventions. In confirmed COVID-19 patients who recover and need a routine bronchoscopy, the correct time to perform it is still unknown; nevertheless, it is considered reasonable to wait at least 30 days from resolution of symptoms, with negative SARS-CoV-2 RNA tests from at least two consecutive NP swab specimens collected 24 hours apart (39). As for evidence showing the rarity of virus that can be cultured in respiratory samples after 9 days after symptom onset, especially in patients with mild diseases (40), that indication is no longer so convincing; consistently with WHO suggestions (41), less rigid local protocols about performing bronchoscopies in patients who have had COVID-19 are acceptable.

\section{Pleural and mediastinal diseases related to COVID-19}

Spontaneous pneumomediastinum (SPM) and pneumothorax (PNX) unrelated to positive pressure ventilation have been recently reported as unusual complications in cases of severe COVID-19 pneumonia (42). Different pathophysiological mechanisms are involved in SPM and PNX, such as diffuse alveolar injury or ischemic parenchymal damage, leading to alveolar rupture and air leak when the intrathoracic pressure increases, for example during persistent coughing (43). Another mechanism is the formation of pneumatoceles or cysts due to COVID-19, even in patients not requiring positive pressure ventilation (44); therefore, barotrauma associated with positive pressure ventilation alone cannot account for the cyst formation, which may contribute to the likelihood of developing a secondary pneumothorax (45-47). It has also been described that the risk of pneumothorax is increased by administration of high dose of methylprednisolone, that probably delays lung healing, as well as the presence of a higher LDH serum peak and peripheral leukocyte count which are expression of a greater lung damage (48). The pathophysiology of SPM is based on the Macklin phenomenon, that is due to a large pressure gradient between the marginal alveoli and the lung interstitium, resulting in air leaks to the surrounding bronchovascular sheath. The damage of the wall of these alveoli can let air escape into the connective tissue sheath, which can result in pulmonary interstitial emphysema, leading to PM (42). Clinical scenarios include COVID-19 patients admitted to hospital with acute pneumothorax, patients who develop PNX or SPM during the hospitalization and patients who develop these complications while intubated and ventilated, either with or without concurrent extracorporeal membrane oxygenation. The diagnosis may be difficult in critically ill patients, but sudden changes in respiratory parameters, acute 
thoracic pain or swelling of the chest and subcutaneous emphysema, should suggest the possibility of pneumothorax or pneumomediastinum (49). SPM in COVID-19 patients usually requires only conservative management (SPM is mostly a self-limiting benign condition) as close monitoring, bed rest, analgesia and oxygen therapy (42). Primary spontaneous PNX could be managed in an outpatients clinic with a conservative approach in minimally symptomatic patients, if local expertise and resources allow it. Otherwise, in symptomatic patients it requires chest drain insertion, in particular in hospitalized patients undergoing invasive or non invasive mechanical ventilation (MV) for ARDS (50). In some selected cases, MV with low pressure support is allowed even if PNX or SPM are present. Sometimes in these patients, according to BTS guidelines, persistent pneumothorax and air leak may ultimately require surgery (51).

\section{Interventional pulmonology procedures in the Covid-19 era}

\section{Bronchoscopy-when to perform}

The COVID-19 pandemic changed how bronchoscopies are performed for all patients. Since a patient undergoing an interventional procedure (for example for a pulmonary nodule) can be asymptomatically infected and he might infect the operators, a safe approach has to be improved in each procedure (52). Indeed, bronchoscopy is one of the procedures at greatest risk of contagion due to aerosols that are generated. If strict prevention rules are not implemented, any infection can be spread from one patient to another, including through healthcare professionals. In areas with a high prevalence, all candidates for bronchoscopy could be potentially carriers of COVID-19. In COVID-19 patients, bronchoscopy has a limited role in the diagnosis and the statements currently published suggest to postpone non-urgent procedures. The Spanish society of pulmonology and thoracic surgery (SEPAR) lists the exceptions to the rule of delaying procedures (immunocompromised patients, atelectasis, hemoptysis, foreign bodies, airway obstruction, help to ventilatory support measure) (53). The British Thoracic Society (BTS) believes that bronchoscopy should be avoided for at least 28 days from onset of infection, if the delay would not be detrimental to the patient prognosis (26). The American Association for Bronchology and Interventional Pulmonology (AABIP) indicate to delay the procedure until the full recovery and infection free declaration of the patient (8); in their view and in the context of suspected or confirmed COVID-19, even urgent/emergent indications should be considered only if a lifesaving bronchoscopic intervention is necessary or if the delay would cause a significant alteration in clinical prognosis. In the case of suspect infection, two negative swabs are suggested.

However, a lot of procedures of interventional pulmonology are requested because of acute or subacute diseases and for the diagnosis (or staging) of cancer. Therefore, one of the main challenges for bronchoscopists during this pandemic is the decision to perform, not to perform or delay procedures. In general, scientific societies proposed strategies focused on the evaluation of the COVID-19 status of patients (infected, suspected, not suspected) and the stratification of exams urgency level. More than ever, indications of every endoscopic request have to be deeply analyzed by the interventional pulmonologist, in discussion with consultants responsible for patients care and/or who requested the procedure. BTS consider the condition of malignancy as the milestone to decide if performing bronchoscopy or not: if the patient doesn't present a malignant (or pre-invasive) lesion, bronchoscopy should be questioned (26). Both AABIP and $\mathrm{SAB}$ present a table as general guidance on procedure urgency. Chest/AABIP Guidelines suggests to postpone not urgent procedures; emergent/urgent bronchoscopies include severe or moderate symptomatic tracheal or bronchial stenosis, symptomatic central airway obstruction (mass or mucus plug), hemoptysis, migrated stents, suspected pulmonary infection in immunocompromised patients, lung nodule, masses, mediastinal or hilar adenopathies suspicious for cancer, foreign object aspiration, alveolar proteinosis requiring whole lung lavage. As regards to suspected or confirmed lung cancer, it is suggested to limit bronchoscopies as follows. For stage I and II, surgical resection should be considered without tissue diagnosis; for stage III with single station N2 disease, lymphonodal staging with linear Endobronchial Ultrasound-guided transbronchial needle aspiration (EBUS-TBNA) or via Transesophageal Bronchoscopic Ultrasound Guided Fine Needle aspiration (EUS-B FNA) is recommended but for patients with bulky/multi-station mediastinal involvement on positron emission tomography (PET), systematic mediastinal staging is not mandatory and PET can be sufficient to define the disease extent in order to minimize HCW exposition; for the same reason, percutaneous biopsy with CT or ultrasound guidance where technically possible are recommended rather than bronchoscopic 
Table 1 Proposal of recommendations for interventional pulmonology procedures during COVID-19 era

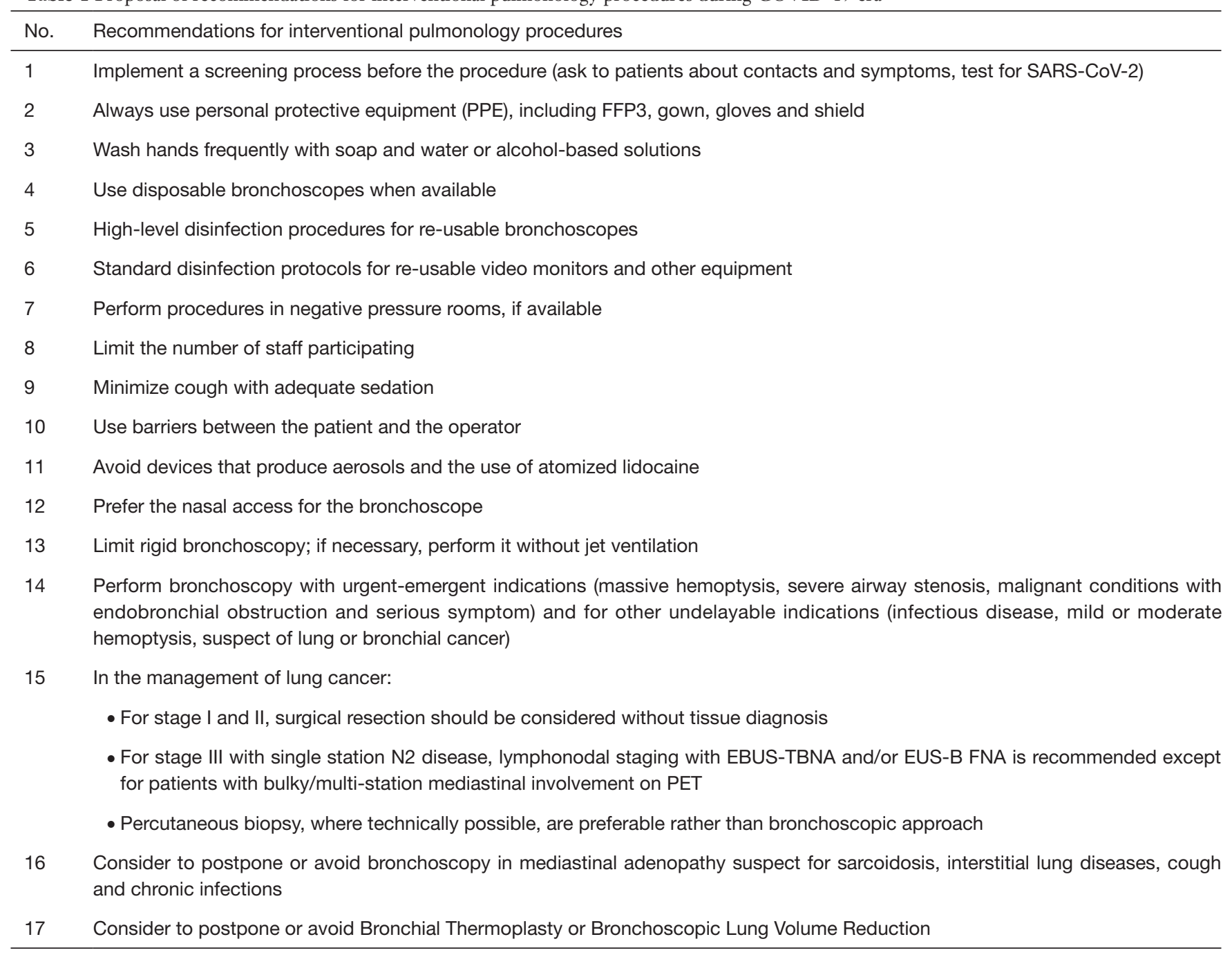

approach (54). Diagnostic and staging procedures cannot be delayed indefinitely because significant delays in therapy (e.g., surgery or radiotherapy) could result in disease progression. Available data suggest that for diagnosis and staging of cancer a brief delay could be implemented without much adverse impact (55); two weeks delay can be considered reasonable in order to contain the risk of SARS-CoV-2 transmission (39). Elective procedures such as bronchoscopic lung volume reduction, bronchial thermoplasty, routine tracheostomy changes and routine surveillance bronchoscopy in transplant patients should be deferred until the epidemic context will improve up to consider safe elective procedures $(8,53)$.

\section{Bronchoscopy—bow to perform}

Performing bronchoscopy in COVID-19 era needs to take into account some recommendations such as those proposed in Table 1. Before scheduling procedures and prior to patient's arrival for planned endoscopic procedures, a screening process should be followed. All the measures suggested by scientific societies and here summarized should be adapted to the possibilities and the resources available in each interventional pulmonology unit; moreover, local epidemiological conditions should be taken into consideration. At the time of scheduling, patients should be asked about contacts and symptoms. If the patient has risk factors, signs or symptoms of a viral infection, the 


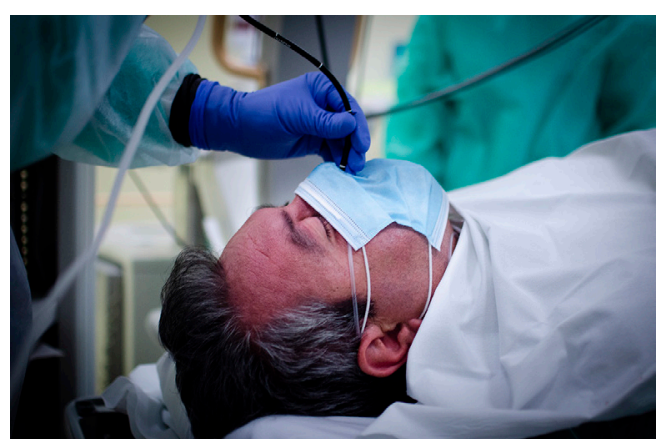

Figure 2 In order to protect the healthcare workers and the environment, the bronchoscope can be passed through a small hole made on a mask worn by the patient.

procedure should be possibly delayed. Prior to performing bronchoscopy in an area where community transmission of SARS-CoV-2 infection is present, it is suggested to test for SARS-CoV-2: if the result is negative, the procedure can be performed (using personal protective equipment, PPE), but if the result is positive non-emergent bronchoscopies should be postponed $(39,52)$. The day before the procedure, all the questions should be asked the patient again. If the patient reports respiratory symptoms due to an unexplained cause, the procedure should be rescheduled and COVID-19 should be investigated. The day of the procedure, both the patient's and caregiver's temperature should be measured and bronchoscopy should be delayed in presence of fever. Same questions about fever, respiratory problems and contacts with ill patients should be posed again. Caregivers are generally prohibited from entering the endoscopic units and they have to stay in a specific waiting area. All societies recommend to limit the number of staff participating in any procedure in order to minimize the use of PPE and reduce known or occult exposure to infectious aerosols. Furthermore, to minimize the potential transmission of the infection it is important to wash hands with soap and water or alcohol-based solutions frequently; specific actions about it should be put in place as it's the most important hygiene measure against cross infections (56). When available, single-use bronchoscopes are to be preferred to standard bronchoscopes $(8,9,57)$. For re-usable bronchoscopes, highlevel disinfection procedures should be performed; standard disinfection protocols are important for re-usable video monitors and other equipment (6). Bronchoscopies should be performed in negative pressure rooms or designated isolation rooms and all members of staff should wear standard Personal Protective Equipment (see paragraph
PPE) $(8,9,26)$. The droplets spread should be minimized using sedation for cough and some barriers between the patient and the operator [e.g., "aerosol box" (58) or masks, see Figure 2]. Devices that produce aerosols and atomized lidocaine should be avoided. Nasal access is to be preferred in flexible bronchoscopy. Rigid bronchoscopy has greater risks, so it should be performed only when necessary and after having reasonably excluded a SARS-CoV-2 infection; in these cases, the procedure should be performed without jet ventilation $(9,30)$.

\section{Management of pleural diseases—when to perform}

Thoracoscopy, pleural biopsies, drainage of pleural effusion or chest drain insertion for pneumothorax could reasonably be considered to be aerosol-generating procedures and recently SARS-CoV-2 viral RNA has been detected in pleural fluid in post-mortem analyses $(59,60)$. Therefore, there is a significant risk of spread of COVID-19 infection if pleural effusion splash during these procedures (61); another possible risk is related to the possible virus aerosolization from an air leak in the chest drain (62). Nevertheless, thoracoscopy together with pleural biopsies and drainage of pleural effusion of suspect malignant origin are considered high priority procedures in the management and treatment of lung cancer; in addition to this, both empyema and abscess evacuation need prompt interventions (63). For these reasons it is essential to stratify procedures according to indication and urgency minimizing hospital access for patients, safeguarding both patients and HCW safety. Guidelines suggest performing diagnostic pleural procedures in patients with suspected pleural infections or malignancy in whom performance status and comorbidities do not preclude systemic anti-cancer therapy. It is also recommended to minimize hospital visits and admission for symptomatic patients with both benign and malignant conditions; admission for talc pleurodesis should be considered after a careful discussion about the risks of hospital admission and alternative strategies, if COVID-19 screening is negative and the procedure can be performed in a non-COVID area. Postponing pleural procedures should be evaluated in patients with malignant pleural disease not considered fit for systemic anti-cancer therapy or in patients with mild or tolerable symptoms; in addition to this, routine follow-up of benign asbestosis or benign pleuritis should be deferred; alternatively, a telephonic or video follow-up should be considered. 


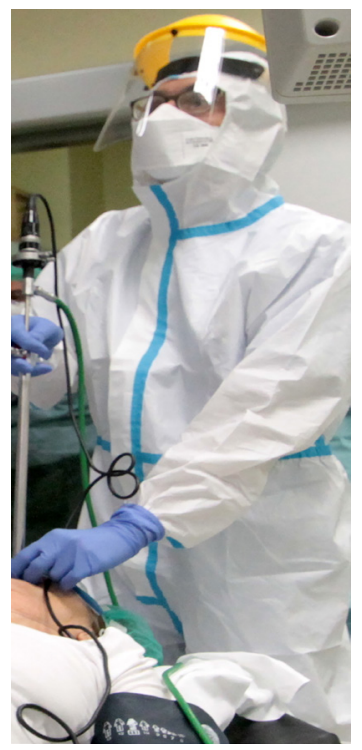

Figure 3 An interventional pulmonologist performs a rigid bronchoscopy correctly wearing PPE: gown, FFP3 mask, eye shield and gloves. PPE, personal protective equipment.

\section{Management of pleural diseases—how to perform}

In suspected pleural infections or malignancy, before planned procedures patients should be investigated about the presence of symptoms consistent with COVID-19 and a nasal or OP swab should be performed within 48 hours; clinicians may also consider a low-dose CT scanning on the day of the procedure to assess radiological evidence of COVID-19 disease (50). During pleural aspirations and chest drain insertion it is suggested to use surgical masks, visors, gowns and gloves; instead, open procedures such as thoracoscopy and indwelling pleural catheter (IPC) insertion should be considered as AGP (50), so HCW should wear a FFP3 mask, a long sleeved gown, gloves and eye protection. Several strategies are suggested to minimize droplets exposure via the chest drain circuit in confirmed or suspected COVID-19 patients: connect any chest drain to wall suction, install a viral filter onto the suction port of a Rocket chest drain bottle or consider the use of digital drain circuits $(51,62)$. Moreover, patients discharged or managed in an outpatient clinic with a pleural device or a chest drain in situ for pneumothorax due to COVID-19, should be advised to self-isolate given the risk, albeit small, of aerosol generation.

\section{PPE}

All staff members participating in endoscopic procedures must use PPE, such as gowns, masks, eye shields and gloves (see Figure 3). When operators wear PPE, their skin should never be exposed, because of the possibility of contact with infected biological fluids. The presence of an observer when a health care worker is putting on (donning) or removing (doffing) PPE could be useful, to identify and immediately correct any mistake: these moments are essential for the right protection of the workers. The use of a checklist is recommended to exactly document the steps in putting on or taking off PPE. Practical training courses about using PPE are very important to increase compliance and improve the correct practice. Using two pairs of gloves is suggested. Gowns should be fluid-resistant or impermeable. Boot coverings should cover the leg up to the midcalf.

Using masks or respirators is crucial; the N95 mask is a particulate filtering facepiece respirator that protects against droplet and airborne transmission of $95 \%$ of particles greater than 0.3 microns in size. Considering the possibility that the availability of materials may be scarce, CDC suggested instructions for prolonged use or reuse of these products (64). Powered air purifying respirators (PAPRs) could have a theoretical benefit over N95 respirators, because they have a higher assigned protection factor (65). However, the reusability of PAPRs may carry risks of contamination if donning and doffing are not correctly done (66); this is why for many reasons there is not a firm conclusion about favoring PAPRs or masks (39). In case of the interruption of protective barriers during a procedure to a patient with suspected or confirmed COVID-19, immediate actions must be taken: the exposed worker should interrupt the procedure immediately and he should be replaced to complete the procedure (backup providers should be available if possible) (8). The right removal of potentially contaminated PPE is the most difficult challenge in preventing inadvertent exposure to pathogens (67). Removal of PPE should take place in a dedicated area that is separate from the endoscopic room or the patient's room. These areas are considered to be contaminated and have to be separated from the clean area used for putting on PPE. Before doffing, the health care worker should disinfect any visible contamination on his PPE and disinfect the outer gloves with an alcohol-based hand rub, waiting for drying. Once removed, all PPE should be discarded in a biohazardous waste container. It is particularly important to avoid contamination of the eyes and mucous membranes when removing facial PPE. The gowns should be taken away from the body and rolled inside-out. Sitting down on a dedicated chair could be useful to remove the boot 
covers. The respirator should be removed touching only the straps, whit a clean pair of gloves. At the end, hands should be disinfected with an alcohol-based hand rub. Every step should be performed without rush, taking the correct amount of time.

\section{Looking forward to the post-pandemic era: the role of interventional pulmonology}

\section{Diagnosis of COVID-19 related fibrosis}

There is growing interest around long term pulmonary complications of SARS-CoV-2 infection, in particular in term of fibrotic damage $(68,69)$. Other coronaviruses have been associated with the development of a fibrotic damage following the acute phase of the disease in a considerable amount of patients (up to 30\%) (70), and it is also known that sub-acute viral infections are involved in the development of fibrosis (71). Moreover, ARDS and prolonged MV, conditions that are frequently found in patients with COVID-19, could be the triggers for the development of such damage $(72,73)$. There are evidences of radiologic pattern changes during the course of the disease (18), but there are still not data on the real incidence of lung fibrosis following the acute phase of the disease. Pathology data are also lacking: in a recent report, the early pathologic changes of the lung parenchyma are described in patients undergoing lobectomy for lung cancer and radiologic and laboratory evidence of SARS-CoV-2 infection, describing the features of organizing pneumonia (OP) and acute fibrinous organizing pneumonia (AFOP) (74). In contrast, other autopsy reports of patients died in ICU due to severe COVID-19 showed the presence of diffuse alveolar damage in the initial and proliferative phase, rarely fibrotic evolution (75). Even if there are growing evidences of the underlying inflammatory pathway leading to the possible development of fibrotic damage (76), on the other hand there are not data on the time of onset of this peculiar evolution: some reports describe fibrotic changes in the acute phase $(77,78)$, while others describe the presence of CT abnormalities at 12 weeks (79). It is also known that some patients exhibit different grades of functional impairment, even after recovery of acute respiratory failure, depending on the severity of the disease and in particular regarding the diffusion lung capacity of carbon monoxide (DLCO) $(80,81)$. The first follow up studies published describe significant abnormalities at lung function tests, CT imaging and the persistence of respiratory and systemic symptoms after several months, depending on the severity of the disease. This data show that longer follow up studies in the post-pandemic period are needed and will probably clarify the real incidence of COVID-19 related fibrosis and the functional impairment of patients hit by this delayed manifestation $(82,83)$. On the other hand, the possible therapeutic implications for the persistent lung abnormalities have been evaluated, focusing in particular on the role of corticosteroids (84); there is also a growing debate over the potential role of antifibrotic therapy for this particular clinical condition (85). However, longer followup are definitely needed.

In this field, bronchoscopy could fill the gap of knowledge with diagnostic techniques used for the diagnosis of diffuse parenchymal lung disease such as transbronchial lung biopsy (TBLB) and transbronchial lung cryobiopsy (TBLC) $(86,87)$. In particular, due to the greater dimension of the samples, TLCB could have an effective role in identifying the pathologic pattern of the COVID-19 related fibrosis. It is well known that, in diseases where a greater tissue sample is required, the gold standard is the surgical lung biopsy (SLB) (86) but, due to the great amount of patients potentially involved and the relatively costeffectiveness of the procedure, compared to the SLB, TLCB could have an important role in the diagnosis of fibrotic sequelae (88). Moreover, understanding the pathological basis of the fibrosis following this disease will have a crucial role in the subsequent therapeutic management. TBLC has already shown to be useful in filling this gap of knowledge in post-mortem analysis: Barisione and co-workers associated pathology patterns found on TBLC samples obtained 30 minutes after death with the lung CT features in patients with SARS-CoV-2-related ARDS $(89,90)$. These data refer to the acute phase of the disease, but this tool could be applicable also in the late phase of the lung damage, once established.

\section{Follow-up in intubated or tracheostomized patients}

As previously said, the disease related to SARS-CoV-2 could originate many different clinical phenotypes (91); in the most severe ones, patients soon develop severe respiratory failure with many typical features of ARDS (92). For this reason, ETI and MV have been largely used in these patients during this pandemic (93); on the other hand, most of the patients undergoing ETI due to this disease needed MV for a long period and consequently the practice of tracheostomy spread through ICUs in order to facilitate the 
weaning process and to reduce the length of ICU stay, even if the improvement in clinical outcomes is not known (94). Actually, tracheostomy can free patients from ventilatory support and, in the peak of pandemic, this is important in order to maximize scarce resources (95). Nevertheless, optimal timing and technique of tracheotomy are not defined (96) and it depends on the availability of resources and have to be decided on a case-by-case basis. Given the great amount of intubated and tracheostomized patients, the next clinical problem is the timing of decannulation. Even if there is still uncertainty in literature on this theme, bronchoscopy could play an important role, along with other clinical tools, in assessing the right time for decannulation and in avoiding decannulation failure $(97,98)$.

ETI and tracheostomy are invasive procedures that can cause also delayed adverse events such as tracheal stenosis or granuloma formation at the end of the tracheal tube (99). The incidence of stenosis ranges up to $20 \%$ of patients undergoing ETI or tracheostomy, and the symptoms usually appear between the 4th and the 8th week after extubation or tracheostomy tube removal (100-102). Moreover, in this particular field, bronchoscopic procedures could cover an important role for the diagnosis and the treatment of postintubation or post-tracheostomy stenosis. Tracheal stenoses have been described in COVID-19 patients after intubation and tracheostomy, considering also that these conditions share a common risk factor: obesity (103). Management of tracheal stenosis is a classical indication for rigid bronchoscopy procedures with the use of various techniques, such as balloon dilatation, laser, argon plasma coagulation, electrocauterization, cryotherapy and stent placement $(104,105)$. Nevertheless, the other therapeutic option is the surgical approach, and it is well defined that a complete and multidisciplinary evaluation of the stenosis between interventional pulmonologists and otolaryngologists is the best way to face these adverse events, considering the characteristics of the stenosis and the consequent prognosis after treatment (104). Given the great number of patients undergoing ETI and tracheostomy, it is quite likely that a large number of tracheal stenosis will be detected in the post-pandemic era (106), with the consequent need for more bronchoscopic procedures in order to diagnose and to treat these affections. Follow up programs for intubated or tracheostomized patients appear to be necessary in order to make a rapid diagnosis and to find the best treatment for each patient, either endoscopic or surgical.

\section{Conclusions}

COVID-19 has become increasingly prevalent worldwide, reaching a pandemic stage in March 2020. This infection is primarily transmitted via droplets and fomites; aerosol transmission is also possible during aerosol-generating procedures.

During the COVID-19 pandemic it is important to minimize the number of interventional pulmonary procedure, balancing risks and rewards for every single procedure in order to reduce community spread of SARS$\mathrm{CoV}-2$ infections and preserve healthcare workforce and hospital resources. Bronchoscopy is an aerosol-generating procedure and for this reason it has to be considered a procedure at high risk of exposure and infection for HCWs and patients.

Even if BAL demonstrated higher sensibility in diagnosis of SARS-CoV-2 infection, compared to sputum or upper respiratory swabs, bronchoscopy should not be a first line testing modality for diagnosis in patients suspected for COVID-19 infection and less invasive tests should be chosen, firstly NP specimen. A tight integration between clinical and laboratory data, analysis of upper respiratory specimens and imaging is essential to establish the correct diagnosis.

Bronchoscopy may be considered in the setting of severe progressive disease after negative upper respiratory specimens or when an alternative diagnosis will change in clinical management. It may also have a role in diagnosing co-infections or in the management of COVID-19 related complications, carefully weighing the benefits and the risks.

It is essential to review the need for each procedure caseby-case in order to stratify and schedule airway procedures according to indication and urgency, postponing non-urgent ones both in COVID-19 or in non-COVID-19 patients.

Each patient who has to undergo bronchoscopy should be investigated for the presence of symptoms suggestive for COVID-19, travels or contacts with a suspicious or confirmed COVID-19 cases and a NP specimen should be obtained. Particularly, in confirmed COVID-19 patients all non-emergent bronchoscopies have to be postponed waiting at least 30 days from resolution of symptoms with at least two consecutive negative NP swabs specimens collected $>24$ hours apart, except for less restrictive local protocols.

If it is necessary to proceed with bronchoscopy is essential to minimize the risk in propagating infection 
performing the procedures in negative pressure rooms or designated isolation room and using appropriate protections for healthcare staff who should be wear contact precautions (face shield, mask, gown and gloves) and N-95 respirators or PAPRs even on asymptomatic patients; proper training on donning and doffing of PPE is crucial to avoid contamination. Limiting the number of staff participating to any procedure, using some kind of barrier between the patient and the operator and using disposable bronchoscopes are also recommended. Rigid bronchoscopy should be avoided when possible.

Thoracoscopy, pleural biopsy and drainage of pleural effusions are not closely aerosol-generating procedures; however, there is a significant risk of spread of SARS-CoV-2 infection linked to possible pleural effusion splash during the procedure; another eventual risk is related to the possible aerosolization of the virus from a chest drain with an air leak.

For these reasons, it is very important to minimize these procedures only to essential ones. Guidelines suggest to continue performing diagnostic pleural procedures in patients with suspected pleural infection or malignancy and who are potentially candidates for systemic anti-cancer therapy; routine follow-up of benign asbestos pleural disease or benign pleuritis should be deferred. Before planned procedures, patients should be investigated about the presence of symptoms consistent with COVID-19 and a nasal or OP swab should be performed within 48 hours.

Utilization of PPE is mandatory; thoracoscopy and IPC insertion should be considered as an AGP so HCWs should wear FFP3 masks, long sleeved gowns, gloves and face shields; during "closed" pleural procedures as aspirations or chest drain insertions it is recommended the use of surgical masks, visors, gowns and gloves.

Finally, a novel potential role for the interventional pulmonology procedures pertains the post pandemic period, in particular regarding the direct and indirect consequences of the viral damage. First, the research on and the diagnosis of COVID-related fibrosis; second, the role of diagnostic and therapeutic bronchoscopy in patients having been intubated and tracheostomized, in order to diagnose, to treat and to avoid the complications related to these invasive procedures (e.g., decannulation failure and post-intubation stenosis).

In conclusion, bronchoscopy should be used sparingly in the evaluation and management of patients with suspected or confirmed COVID-19 infections. A careful review of the need for every single procedure postponing non-urgent ones and the use of PPE are the main strategies to minimize the risk of exposure and infection for health-care workers and patients.

\section{Acknowledgments}

The authors acknowledge the nurses of the Interventional Pulmonology Unit of Reggio Emilia for their passionate work in favor of the patients.

Funding: None.

\section{Footnote}

Reporting Checklist: The authors have completed the Narrative Review reporting checklist. Available at http:// dx.doi.org/10.21037/jtd-20-2192

Peer Review File: Available at http://dx.doi.org/10.21037/jtd20-2192

Conflicts of Interest: All authors have completed the ICMJE uniform disclosure form (available at http://dx.doi. org/10.21037/jtd-20-2192). The authors have no conflicts of interest to declare.

Ethical Statement: The authors are accountable for all aspects of the work in ensuring that questions related to the accuracy or integrity of any part of the work are appropriately investigated and resolved. Written informed consent was obtained from people in the pictures (Figures 2,3).

Open Access Statement: This is an Open Access article distributed in accordance with the Creative Commons Attribution-NonCommercial-NoDerivs 4.0 International License (CC BY-NC-ND 4.0), which permits the noncommercial replication and distribution of the article with the strict proviso that no changes or edits are made and the original work is properly cited (including links to both the formal publication through the relevant DOI and the license). See: https://creativecommons.org/licenses/by-nc-nd/4.0/.

\section{References}

1. Li Q, Guan X, Wu P, et al. Early transmission dynamics in Wuhan, China, of novel coronavirus-infected pneumonia. N Engl J Med 2020;382:1199-207.

2. Lu R, Zhao X, Li J, et al. Genomic characterisation and epidemiology of 2019 novel coronavirus: implications for virus origins and receptor binding. Lancet 2020;395:565-74. 
3. Rothe C, Schunk M, Sothmann P, et al. Transmission of 2019-NCOV infection from an asymptomatic contact in Germany. N Engl J Med 2020;382:970-1.

4. WHO. Coronavirus disease (COVID-19) Situation Report - 115 Data as received by WHO from national authorities by 10:00 CEST, 14 May 2020.

5. Guan WJ, Ni ZY, Hu Y, et al. Clinical Characteristics of Coronavirus Disease 2019 in China. N Engl J Med 2020;382:1708-20.

6. World Health Organization. WHO Clinical management of severe acute respiratory infection (SARI) when COVID-19 disease is suspected. WHO 2020;2019:12.

7. Group of Interventional Respiratory Medicine, Chinese Thoracic Society. Expert consensus for bronchoscopy during the epidemic of 2019 novel coronavirus infection (Trial version). Zhonghua Jie He He Hu Xi Za Zhi 2020;43:199-202.

8. Wahidi MM, Lamb C, Murgu S, et al. American Association for Bronchology and Interventional Pulmonology (AABIP) Statement on the Use of Bronchoscopy and Respiratory Specimen Collection in Patients With Suspected or Confirmed COVID-19 Infection. J Bronchology Interv Pulmonol 2020;27:e52-4.

9. Pritchett MA, Oberg CL, Belanger A, et al. Society for Advanced Bronchoscopy Consensus Statement and Guidelines for bronchoscopy and airway management amid the COVID-19 pandemic Society for Advanced Bronchoscopy Consensus Statement and Guidelines for bronchoscopy and airway management amid the COV.J Thorac Dis 2020;12:1781-98.

10. Gaunt ER, Hardie A, Claas ECJ, et al. Epidemiology and clinical presentations of the four human coronaviruses 229E, HKU1, NL63, and OC43 detected over 3 years using a novel multiplex real-time PCR method. J Clin Microbiol 2010;48:2940-7.

11. Charlton CL, Babady E, Ginocchio CC, et al. Practical Guidance for Clinical Microbiology Laboratories: Viruses Causing Acute Respiratory Tract Infections. Clin Microbiol Rev 2018;32:e00042-18.

12. Falsey AR, Formica MA, Walsh EE. Simple method for combining sputum and nasal samples for virus detection by reverse transcriptase PCR. J Clin Microbiol 2012;50:2835.

13. Wang W, Xu Y, Gao R, et al. Detection of SARSCoV-2 in Different Types of Clinical Specimens. JAMA 2020;323:1843-4.

14. Scohy A, Anantharajah A, Bodéus M, et al. Low performance of rapid antigen detection test as frontline testing for COVID-19 diagnosis. J Clin Virol
2020;129:104455.

15. Dinnes J, Deeks JJ, Adriano A, et al. Rapid, point-ofcare antigen and molecular-based tests for diagnosis of SARS-CoV-2 infection. Cochrane Database Syst Rev 2021;3:CD013705.

16. Bernheim A, Mei X, Huang M, et al. Chest CT Findings in Coronavirus Disease-19 (COVID-19): Relationship to Duration of Infection. Radiology 2020;295:200463.

17. Fang Y, Zhang H, Xie J, et al. Sensitivity of Chest CT for COVID-19: Comparison to RT-PCR. Radiology 2020;296:E115-7.

18. Liang T, Liu Z, Wu CC, et al. Evolution of CT findings in patients with mild COVID-19 pneumonia. Eur Radiol 2020;30:4865-73

19. Simpson S, Kay FU, Abbara S, et al. Radiological Society of North America Expert Consensus Statement on Reporting Chest CT Findings Related to COVID-19. Endorsed by the Society of Thoracic Radiology, the American College of Radiology, and RSNA. Radiol Cardiothorac Imaging 2020;2:e200152.

20. Ai T, Yang Z, Hou H, et al. Correlation of Chest CT and RT-PCR Testing in Coronavirus Disease 2019 (COVID-19) in China: A Report of 1014 Cases. Radiology 2020;296:E32-40.

21. Rodrigues JCL, Hare SS, Edey A, et al. An update on COVID-19 for the radiologist - A British society of Thoracic Imaging statement. Clinical Radiology 2020;75:323-5.

22. Centers for Disease Control and Prevention. Testing for COVID-19. Available online: https://www.cdc.gov/ coronavirus/2019-ncov/symptoms-testing/testing.html

23. Zhang W, Du RH, Li B, et al. Molecular and serological investigation of 2019-nCoV infected patients: implication of multiple shedding routes. Emerg Microbes Infect 2020;9:386-9.

24. Hoffman T, Nissen K, Krambrich J, et al. Evaluation of a COVID-19 IgM and IgG rapid test; an efficient tool for assessment of past exposure to SARS-CoV-2. Infect Ecol Epidemiol 2020;10:1754538.

25. Facciolongo N, Piro R, Menzella F, et al. Training and practice in bronchoscopy: A national survey in Italy. Monaldi Arch Chest Dis - Pulm Ser 2013;79:128-33.

26. DR Baldwin, WS Lim, R Rintoul, et al. Recommendations for day case bronchoscopy services during the COVID-19 pandemic Version 2: Services during the restoration and recovery COVID-19 endemic phase. Br Thorac Soc 2020.

27. Geri P, Salton F, Zuccatosta L, et al. Limited role for bronchoalveolar lavage to exclude COVID-19 after 
negative upper respiratory tract swabs: a multicentre study. Eur Respir J 2020;56:2001733.

28. Luo F, Darwiche K, Singh S, et al. Performing Bronchoscopy in Times of the COVID-19 Pandemic: Practice Statement from an International Expert Panel. Respiration 2020;99:417-22.

29. Van Doremalen N, Bushmaker T, Morris DH, et al. Aerosol and surface stability of SARS-CoV-2 as compared with SARS-CoV-1. N Engl J Med 2020;382:1564-7.

30. AIPO-ITS. Ruolo e modalità di esecuzione della broncoscopia nella pandemia da COVID-19 - Position Paper AIPO - ITS. Available online: http://www.aiponet. it/news/speciale-covid-19/2456-ruolo-e-modalita-diesecuzione-della-broncoscopia-nella-pandemia-da-covid19-position-paper.html

31. Livrieri F, Ghidoni G, Piro R, et al. May 2020: Is It Always COVID-19 No Matter What? Int Med Case Rep J 2020;13:563-7.

32. Kollias A, Kyriakoulis KG, Dimakakos E, et al. Thromboembolic risk and anticoagulant therapy in COVID-19 patients: emerging evidence and call for action. Br J Haematol 2020;189:846-7.

33. Bikdeli B, Madhavan M V., Jimenez D, et al. COVID-19 and Thrombotic or Thromboembolic Disease:

Implications for Prevention, Antithrombotic Therapy, and Follow-Up: JACC State-of-the-Art Review. J Am Coll Cardiol 2020;75:2950-73.

34. Singh RK, Saran S, Baronia AK. The practice of tracheostomy decannulation-a systematic review. J Intensive Care 2017;5:38

35. de Medeiros GC, Sassi FC, Lirani-Silva C, et al. Criteria for tracheostomy decannulation: literature review. Codas 2019;31:e20180228.

36. Koehler P, Bassetti M, Chakrabarti A, et al. Defining and managing COVID-19-associated pulmonary aspergillosis: the $2020 \mathrm{ECMM} / \mathrm{ISHAM}$ consensus criteria for research and clinical guidance. Lancet Infect Dis 2020:S14733099(20)30847-1

37. Alanio A, Dellière S, Fodil S, et al. Prevalence of putative invasive pulmonary aspergillosis in critically ill patients with COVID-19. Lancet Respir Med 2020;8:e48-9.

38. van Arkel ALE, Rijpstra TA, Belderbos HNA, et al. COVID-19-associated Pulmonary Aspergillosis. Am J Respir Crit Care Med 2020;202:132-5.

39. Wahidi MM, Shojaee S, Lamb CR, et al. The Use of Bronchoscopy During the Coronavirus Disease 2019 Pandemic: CHEST/AABIP Guideline and Expert Panel Report. Chest 2020;158:1268-81.
40. Bullard J, Dust K, Funk D, et al. redicting Infectious Severe Acute Respiratory Syndrome Coronavirus 2 From Diagnostic Samples. Clin Infect Dis 2020;71:2663-6.

41. World Health Organization. Criteria for releasing COVID-19 patients from isolation. Available online: https://www.who.int/news-room/commentaries/detail/ criteria-for-releasing-covid-19-patients-from-isolation

42. Elhakim TS, Abdul HS, Pelaez Romero C, et al. Spontaneous pneumomediastinum, pneumothorax and subcutaneous emphysema in COVID-19 pneumonia: a rare case and literature review. BMJ Case Rep 2020;13:e239489.

43. Joynt GM, Antonio GE, Lam P, et al. Late-stage adult respiratory distress syndrome caused by severe acute respiratory syndrome: abnormal findings at thin-section CT. Radiology 2004;230:339-46.

44. Hameed M, Jamal W, Yousaf M, et al. Pneumothorax In Covid-19 Pneumonia: A case series. Respir Med Case Rep 2020;31:101265.

45. Zhou C, Gao C, Xie Y, et al. COVID-19 with spontaneous pneumomediastinum. Lancet Infect Dis 2020;20:510.

46. Liu K, Zeng Y, Xie P, et al. COVID-19 with cystic features on computed tomography: A case report. Medicine (Baltimore) 2020;99:e20175.

47. Sun R, Liu H, Wang X. Mediastinal Emphysema, Giant Bulla, and Pneumothorax Developed during the Course of COVID-19 Pneumonia. Korean J Radiol 2020;21:541-4.

48. Sihoe ADL, Wong RHL, Lee ATH, et al. Severe acute respiratory syndrome complicated by spontaneous pneumothorax. Chest 2004;125:2345-51.

49. Ceylan KC, Batihan G, Yazgan S, et al. Pleural complications in patients with coronavirus disease 2019 (COVID-19): how to safely apply and follow-up with a chest tube during the pandemic? Eur J Cardiothorac Surg 2020;58:1216-21.

50. British Thoracic Society. Pleural services during the COVID-19 Pandemic. British Thoracic Society 2020;1-4.

51. MacDuff A, Arnold A, Harvey J. Management of spontaneous pneumothorax: British Thoracic Society Pleural Disease Guideline 2010. Thorax 2010;65 Suppl 2:ii18-31.

52. Ost DE. Bronchoscopy in the Age of COVID-19. J Bronchology Interv Pulmonol 2020;27:160-2.

53. Cordovilla R, Álvarez S, Llanos L, et al. SEPAR and AEER consensus recommendations on the Use of Bronchoscopy and Airway Sampling in Patients with Suspected or Confirmed COVID-19 Infection. Arch Bronconeumol 2020;56 Suppl 2:19-26.

54. Steinfort DP, Herth FJF, Irving LB, et al. Safe performance 
of diagnostic bronchoscopy/EBUS during the SARS-

CoV-2 pandemic. Respirology 2020;25:703-8.

55. Ost DE, Ernst A, Lei X, et al. Diagnostic Yield and Complications of Bronchoscopy for Peripheral Lung Lesions. Results of the AQuIRE Registry. Am J Respir Crit Care Med 2016;193:68-77.

56. West R, Michie S, Rubin GJ, et al. Applying principles of behaviour change to reduce SARS-CoV-2 transmission.

Nat Hum Behav 2020;4:451-9.

57. Lentz RJ, Colt H. Summarizing societal guidelines regarding bronchoscopy during the COVID-19 pandemic. Respirology 2020;25:574-7.

58. Saito T, Asai T. Aerosol containment device for airway management of patients with COVID-19: a narrative review. J Anesth 2020;23:1-6.

59. Pieracci FM, Burlew CC, Spain D, et al. Tube thoracostomy during the COVID-19 pandemic: guidance and recommendations from the AAST Acute Care Surgery and Critical Care Committees. Trauma Surg Acute Care Open 2020;5:e000498.

60. Schaller T, Hirschbühl K, Burkhardt K, et al. Postmortem Examination of Patients With COVID-19. JAMA 2020;323:2518-20.

61. Dhanasopon AP, Zurich H, Preda A. Chest Tube Drainage in the Age of COVID-19. Physician Assist Clin 2021;6:261-5.

62. Bilkhu R, Viviano A, Saftic I, et al. COVID-19: Chest Drains With Air Leak - The Silent 'Super Spreader'? April 2020. doi:10.25373/ctsnet. 12089130

63. Catanese S, Pentheroudakis G, Douillard J-Y, et al. ESMO Management and treatment adapted recommendations in the COVID-19 era: Pancreatic Cancer. ESMO Open 2020;5:e000804.

64. Center for Disease Control and Prevention. Strategies to optimize the supply of PPE and equipment. Available online: https://apic.org/advocacy_update/strategies-tooptimize-the-supply-of-ppe-and-equipment/

65. OSHA. Assigned protection factors for the revised respiratory protection standard. Osha 2009;1-47.

66. Zamora JE, Murdoch J, Simchison B, et al. Contamination: A comparison of 2 personal protective systems. CMAJ 2006;175:249-54.

67. Ortega R, Bhadelia N, Obanor O, et al. Putting on and removing personal protective equipment. $\mathrm{N}$ Engl J Med 2015;372:e16.

68. Spagnolo P, Balestro E, Aliberti S, et al. Pulmonary fibrosis secondary to COVID-19: a call to arms? Lancet Respir Med 2020;8:750-2.
69. Scelfo C, Fontana M, Casalini E, et al. A Dangerous Consequence of the Recent Pandemic: Early Lung Fibrosis Following COVID-19 Pneumonia - Case Reports. Ther Clin Risk Manag 2020;16:1039-46.

70. Das KM, Lee EY, Singh R, et al. Follow-up chest radiographic findings in patients with MERS-CoV after recovery. Indian J Radiol Imaging 2017;27:342-9.

71. Sheng G, Chen P, Wei Y, et al. Viral Infection Increases the Risk of Idiopathic Pulmonary Fibrosis: A MetaAnalysis. Chest 2020;157:1175-87.

72. Matthay MA, Zemans RL, Zimmerman GA, et al. Acute respiratory distress syndrome. Nat Rev Dis Primers 2019;5:18.

73. Cabrera-Benitez NE, Laffey JG, Parotto M, et al. Mechanical ventilation-associated lung fibrosis in acute respiratory distress syndrome: A significant contributor to poor outcome. Anesthesiology 2014;121:189-98.

74. Tian S, Hu W, Niu L, et al. Pulmonary Pathology of Early-Phase 2019 Novel Coronavirus (COVID-19) Pneumonia in Two Patients With Lung Cancer. J Thorac Oncol 2020;15:700-4.

75. Carsana L, Sonzogni A, Nasr A, et al. Pulmonary postmortem findings in a large series of COVID-19 cases from Northern Italy. medRxiv 2020;2020.04.19.20054262.

76. Wang J, Wang BJ, Yang JC, et al. Advances in the research of mechanism of pulmonary fibrosis induced by Corona Virus Disease 2019 and the corresponding therapeutic measures. Zhonghua Shao Shang Za Zhi 2020;36:E006.

77. Schwensen HF, Borreschmidt LK, Storgaard M, et al. Fatal pulmonary fibrosis: a post-COVID-19 autopsy case. J Clin Pathol 2020. [Epub ahead of print]. doi: 10.1136/ jclinpath-2020-206879.

78. Combet M, Pavot A, Savale L, et al. Rapid onset honeycombing fibrosis in spontaneously breathing patient with COVID-19. Eur Respir J 2020;56:2001808.

79. Shah AS, Wong AW, Hague CJ, et al. A prospective study of 12-week respiratory outcomes in COVID-19-related hospitalisations. Thorax 2020. [Epub ahead of print]. doi: 10.1136/thoraxjnl-2020-216308.

80. Mo X, Jian W, Su Z, et al. Abnormal pulmonary function in COVID-19 patients at time of hospital discharge. Eur Respir J 2020;55:2001217.

81. Frija-Masson J, Debray MP, Gilbert M, et al. Functional characteristics of patients with SARS-CoV-2 pneumonia at 30 days post-infection. Eur Respir J 2020;56:2001754.

82. Huang C, Huang L, Wang Y, et al. 6-month consequences of COVID-19 in patients discharged from hospital: a cohort study. Lancet 2021;397:220-32. 
83. Guler SA, Ebner L, Beigelman C, et al. Pulmonary function and radiological features four months after COVID-19: first results from the national prospective observational Swiss COVID-19 lung study. Eur Respir J 2021;2003690.

84. Myall KJ, Mukherjee B, Castanheira AM, et al. Persistent Post-COVID-19 Inflammatory Interstitial Lung Disease: An Observational Study of Corticosteroid Treatment. Ann Am Thorac Soc 2021. [Epub ahead of print]. doi: 10.1513/ AnnalsATS.202008-1002OC.

85. George PM, Wells AU, Jenkins RG. Pulmonary fibrosis and COVID-19: the potential role for antifibrotic therapy. Lancet Respir Med 2020;8:807-15.

86. Bradley B, Branley HM, Egan JJ, et al. Interstitial lung disease guideline: the British Thoracic Society in collaboration with the Thoracic Society of Australia and New Zealand and the Irish Thoracic Society. Thorax 2008;63 Suppl 5:v1-58.

87. Maldonado F, Danoff SK, Wells AU, et al. Transbronchial Cryobiopsy for the Diagnosis of Interstitial Lung Diseases: CHEST Guideline and Expert Panel Report. Chest 2020;157:1030-42.

88. Hernández-González F, Lucena CM, Ramírez J, et al. Cryobiopsy in the Diagnosis of Diffuse Interstitial Lung Disease: Yield and Cost-Effectiveness Analysis. Arch Bronconeumol 2015;51:261-7.

89. Grillo F, Barisione E, Ball L, et al. Lung fibrosis: an undervalued finding in COVID-19 pathological series. Lancet Infect Dis 2021;21:e72.

90. Barisione E, Grillo F, Ball L, et al. Fibrotic progression and radiologic correlation in matched lung samples from COVID-19 post-mortems. Virchows Arch 2021;478:471-85.

91. Huang C, Wang Y, Li X, et al. Clinical features of patients infected with 2019 novel coronavirus in Wuhan, China. Lancet 2020;395:497-506.

92. Ranieri VM, Rubenfeld GD, Thompson BT, et al. Acute respiratory distress syndrome: The Berlin definition. JAMA 2012;307:2526-33.

93. Grasselli G, Zangrillo A, Zanella A, et al. Baseline Characteristics and Outcomes of 1591 Patients Infected with SARS-CoV-2 Admitted to ICUs of the Lombardy Region, Italy. JAMA 2020;323:1574-81.

94. Mattioli F, Fermi M, Ghirelli M, et al. Tracheostomy in the COVID-19 pandemic. Eur Arch Otorhinolaryngol 2020;277:2133-5.

95. McGrath BA, Brenner MJ, Warrillow SJ, et al. Tracheostomy in the COVID-19 era: global and multidisciplinary guidance. Lancet Respir Med 2020;8:717-25.
96. Botti C, Lusetti F, Peroni S, et al. The Role of Tracheotomy and Timing of Weaning and Decannulation in Patients Affected by Severe COVID-19. Ear Nose Throat J 2021;100:116S-119S.

97. Kutsukutsa J, Kuupiel D, Monori-Kiss A, et al. Tracheostomy decannulation methods and procedures for assessing readiness for decannulation in adults: a systematic scoping review. Int J Evid Based Healthc 2019;17:74-91.

98. Lee TS, Wu Y. Bedside fiberoptic bronchoscopy for tracheostomy decannulation. Respir Med 1995;89:571-5.

99. Cooper JD. Tracheal Injuries Complicating Prolonged Intubation and Tracheostomy. Thorac Surg Clin 2018;28:139-44.

100. Sarper A, Ayten A, Eser I, et al. Tracheal stenosis after tracheostomy or intubation: Review with special regard to cause and management. Tex Heart Inst J 2005;32:154-8.

101. Farzanegan R, Farzanegan B, Zangi M, et al. Incidence Rate of Post-Intubation Tracheal Stenosis in Patients Admitted to Five Intensive Care Units in Iran. Iran Red Crescent Med J 2016;18:e37574.

102. Esteller-Moré E, Ibañez J, Matiñó E, et al. Prognostic factors in laryngotracheal injury following intubation and/or tracheotomy in ICU patients. Eur Arch Otorhinolaryngol 2005;262:880-3.

103. Mattioli F, Marchioni A, Andreani A, et al. Post-intubation tracheal stenosis in COVID-19 patients. Eur Arch Otorhinolaryngol 2021;278:847-8.

104. Galluccio G, Lucantoni G, Battistoni P, et al. Interventional endoscopy in the management of benign tracheal stenoses: definitive treatment at long-term followup. Eur J Cardiothorac Surg 2009;35:429-33.

105. Freitas C, Martins N, Novais-Bastos H, et al. The role of interventional bronchoscopy in the management of post-intubation tracheal stenosis: A 20-year experience. Pulmonology 2019. [Epub ahead of print]. doi: 10.1016/ j.pulmoe.2019.12.004.

106. Piazza C, Filauro M, Dikkers FG, et al. Long-term intubation and high rate of tracheostomy in COVID-19 patients might determine an unprecedented increase of airway stenoses: a call to action from the European Laryngological Society. Eur Arch Otorhinolaryngol 2021;278:1-7.

Cite this article as: Piro R, Casalini E, Livrieri F, Fontana M, Ghidoni G, Taddei S, Facciolongo N. Interventional pulmonology during COVID-19 pandemic: current evidence and future perspectives. J Thorac Dis 2021;13(4):2495-2509. doi: $10.21037 /$ jtd-20-2192 